\title{
Details of the Non-Unitarity Proof for Highest Weight Representations of the Virasoro Algebra
}

\author{
Daniel Friedan ${ }^{1}$, Zongan Qiu $^{2}$, and Stephen Shenker ${ }^{1}$ \\ ${ }^{1}$ Enrico Fermi and James Franck Institutes, and Department of Physics, University of Chicago, \\ Chicago, II 60637, USA \\ ${ }^{2}$ Institute for Advanced Studies, Princeton, NJ 08540, USA
}

\begin{abstract}
We give an exposition of the details of the proof that all highest weight representations of the Virasoro algebra for $c<1$ which are not in the discrete series are non-unitary.
\end{abstract}

The Virasoro algebra is the infinite dimensional Lie algebra with generators $L_{n}$, $n \in \mathbb{Z}$, satisfying the commutation relations

$$
\left[L_{m}, L_{n}\right]=(m-n) L_{m+n}+\frac{1}{12} c\left(m^{3}-m\right) \delta_{m+n, 0} .
$$

The number $c$ is called the central charge. The Verma module $V(c, h)$ is the representation of the Virasoro algebra generated by a vector $|h\rangle$ satisfying

$$
L_{0}|h\rangle=h|h\rangle, \quad L_{n}|h\rangle=0, \quad n>0,
$$

and spanned by the linearly independent vectors $|h\rangle$ and

$$
L_{-k_{1}} L_{-k_{2}} \ldots L_{-k_{n}}|h\rangle, \quad 1 \leqq k_{1} \leqq k_{2} \leqq \ldots \leqq k_{n} .
$$

We assume that both $c$ and $h$ are real. In this case, a hermitian inner product on $V(c, h)$ is defined by $\langle h \mid h\rangle=1$, and $L_{n}^{\dagger}=L_{-n}$. Define, for $p$ and $q$ positive integers,

$$
c(m)=1-\frac{6}{m(m+1)}, \quad h_{p, q}(m)=\frac{((m+1) p-m q)^{2}-1}{4 m(m+1)} .
$$

The non-unitary theorem [1] is

Theorem 1. For $c<1$ there are negative metric states in $V(c, h)$ if $(c, h)$ does not belong to the discrete list

$$
c=c(m), \quad m=2,3,4 \ldots, \quad h=h_{p, q}(m), \quad p+q \leqq m .
$$

This work was supported in part by DOE grant DE-FG02-84ER-45144, NSF grant PHY-8451285 and the Sloan Foundation 
The proof of Theorem 1 was given in [1]. The present paper is an exposition of the details of that proof. We recommend the graphs in [1] as a visual aid.

There are analogous non-unitarity theorems for the $N=1$ supersymmetric extensions of the Virasoro algebra [1,2]. The details of the proofs of the $N=1$ nonunitarity theorems are exactly parallel to the proof of the Virasoro theorem. Goddard et al. [3] proved that all representations in the discrete series allowed by the non-unitarity theorems for the Virasoro algebra and its $N=1$ extensions are in fact unitary. Boucher et al. [4] have given the non-unitarity theorems for the $N=2$ extensions. The $N=2$ proofs [5] are somewhat different from the $N<2$ proofs. Di Vecchia, Petersen, $\mathrm{Yu}$, and Zheng have proved that the discrete series of representations allowed by the $N=2$ non-unitarity theorems are in fact unitary [6].

For $N$ a nonnegative integer, define level $N$ to be the eigenspace of the Verma module on which $L_{0}$ has eigenvalue $h+N$. Level 0 is spanned by $|h\rangle$, and level $N$, $N \geqq 1$, is spanned by the vectors listed in (3) which satisfy $\sum k_{i}=N$. Level $N$ has dimension $P(N)$, the partition number of $N$. Clearly, the levels span $V(c, h)$ and are linearly independent. Since $L_{0}^{\dagger}=L_{0}$, levels $N$ and $N^{\prime}$ are orthogonal if $N \neq N^{\prime}$. Define the null subspace on level $N$ to be the subspace of vectors in level $N$ which are orthogonal to all of level $N$, and thus to all of $V(c, h)$.

The inner products of the states on level $N$ listed in (3) form a $P(N) \times P(N)$ real symmetric matrix $M_{N}(c, h)$ whose entries are polynomials in $c$ and $h$. An explicit formula for the determinant of this matrix was announced by $\mathrm{Kac}$ [7] and proved by Feigin and Fuchs [8]. Up to multiplication by a positive number independent of $c$ and $h$,

$$
\operatorname{det} M_{N}(c, h)=\prod_{\substack{p, q \geqq 1 \\ p q \leqq N}}\left(h-h_{p, q}(m)\right)^{P(N-p q)},
$$

where $h_{p, q}(m)$ is given by Eq. (4). In Eq. (6) it does not matter which branch is chosen for $m$ as a function of $c$. For $c<1$ we choose by convention the branch $0<m<\infty$. There is a nontrivial null subspace on level $N$ if and only if $\operatorname{det} M_{N}(c, h)=0$.

$\mathrm{Kac}$ [9] showed that, for $c \geqq 1$, the metric on $V(c, h)$ is nonnegative if and only if $h \geqq 0$. Direct calculation gives the $1 \times 1$ matrix $M_{1}=2 h$, so $h \geqq 0$ is necessary if the metric is to be nonnegative. It is straightforward to verify that, in the limit $h \rightarrow+\infty$, $M_{N}$ goes to a diagonal matrix with positive entries. It is also straightforward to check that $\operatorname{det} M_{N}(c, h) \neq 0$ for $c>1, h>0$. Therefore $M_{N}(c, h)$ is nondegenerate and positive for $c>1, h>0$, and is non-negative for $c \geqq 1, h \geqq 0$. Since this is true for all levels $N$, the result follows.

The proof of Theorem 1 is entirely elementary. The strategy is to consider the matrices $M_{N}, N=1,2, \ldots$, one by one. For each $N$ we find a subset $G_{N}$ of the halfplane $c<1$ on which $M_{N}(c, h)$ has a negative eigenvalue. We then say that the subset $G_{N}$ has been eliminated. Theorem 1 will follow from the fact that the discrete set (5) is the complement of $\bigcup_{N} G_{N}$ in the half-plane $c<1$.

Henceforth we write $h_{p, q}(c)$ in place of $h_{p, q}(m)$, with the understanding that, for $c<1$, we choose the branch of $m$ with $0<m<\infty$. Write $C_{p, q}$ for the vanishing curve $h=h_{p, q}(c)$. Because $\operatorname{det} M_{N}(c, h)$ vanishes on the curve $C_{p, q}$ for $p q \leqq N$, we say that 
the vanishing curve $C_{p, q}$ first appears on level $p q$, and that the vanishing curves on level $N$ are the $C_{p, q}, p q \leqq N$. The curve $C_{p, q}$ intersects the line $c=1$ at $h=h_{p, q}(1)$ $=(p-q)^{2} / 4$. Orient each vanishing curve so that $c=1$ is the initial point, and forward is the direction of decreasing $c$.

Proposition 1. When the curve $C_{p, 1}$ first appears on level $N=p$, it intersects no other vanishing curves in the half-plane $c<1$. When $C_{p, q}, q>1$, first appears on level $N=p q$, its first intersection, moving forward from $c=1$, is with $C_{q-1, p}$ at $m=p+q-1$.

Proof. The proof is straightforward algebra.

For $q=1$ define $C_{p, 1}^{\prime}$ to be all of $C_{p, q}$ in the half-plane $c<1$. For $q>1$ define $C_{p, q}^{\prime}$ to be the part of $C_{p, q}$ for which $m>p+q-1$. That is, $C_{p, q}^{\prime}$ is the open subset of $C_{p, q}$ between $c=1$ and the first intersection of $C_{p, q}$ on level $N=p q$. The first step in the proof of Theorem 1 is to eliminate all of the half-plane $c<1$ except the curves $C_{p, q}^{\prime}$. For $N \geqq 1$ define

$$
S_{N}=\bigcup_{q<p, p q \leqq N}\left\{(c, h): c<1, h_{q, p}(c) \leqq h \leqq h_{p, q}(c)\right\} \bigcup_{p^{2} \leqq N}\left\{(c, h): c<1, h \leqq h_{p, p}(c)\right\} .
$$

Proposition 2. $\lim _{N \rightarrow \infty} S_{N}$ is the half-plane $c<1$.

Define a first intersection $F$ on $C_{p, q}^{\prime}$ to be an intersection of $C_{p, q}^{\prime}$ and $C_{p^{\prime}, q^{\prime}}$, $p^{\prime} q^{\prime}>p q$, such that, on level $N^{\prime}=p^{\prime} q^{\prime},(c, h)$ is the first intersection encountered on $C_{p, q}^{\prime}$, starting from $c=1$.

Proposition 3. The first intersections on $C_{p, q}^{\prime}$ are the intersections $F_{p, q, k}$ of $C_{p, q}^{\prime}$ and $C_{p^{\prime}, q^{\prime}}=C_{q+k-1, p+k}, k \geqq 1 . F_{p, q, k}$ is the point $h=h_{p, q}(m), m=p+q+k-1$. Each of these first intersections is, at level $p^{\prime} q^{\prime}$, the intersection of exactly two vanishing curves.

Proof. The proof is straightforward algebra.

It immediately follows that

Proposition 4. The discrete list (5) consists exactly of the first intersections, on all the vanishing curves $C_{p, q}^{\prime}$.

Define $R_{1,1}$ to be the open quadrant $c<1, h<0$. Define $R_{p, 1}=R_{1, p}$, for $p>1$, to be the open region bounded by $C_{p, 1}^{\prime}, C_{p-1,1}^{\prime}$, and $C_{1, p}^{\prime}$. For $p, q>1$, define $R_{p, q}$ to be the open region bounded by $C_{p, q}^{\prime}, C_{p-1, q-1}^{\prime}$, and $C_{q-1, p}^{\prime}$.

Proposition 5. No vanishing curves on level $N=p q$ intersect $R_{p, q}$.

Proof. A vanishing curve which did intersect $R_{p, q}$ would have to intersect its boundary. By Proposition 3, this does not happen.

Proposition 6. $S_{N}-S_{N-1}=\bigcup_{p q=N} R_{p, q} \bigcup_{p q=N} C_{p, q}^{\prime}$.

Proposition 7. Except possibly for the curves $C_{p, q}^{\prime}, p q \leqq N$, all of $S_{N}$ is eliminated on levels $\leqq N$. 
Proof. The proof is by induction in $N$. The proposition is clearly true for $N=1$, because $S_{1}$ is the quadrant $c<1, h \leqq 0$, and $C_{1,1}^{\prime}$ is the line $h=0, c<1$. Now suppose the proposition is true for $N-1$. We show that it is also true for $N$. By Proposition 6, we need to show that the $R_{p, q}, p q=N$, are eliminated on level $N$.

We say that two connected regions of the $(c, h)$ plane are contiguous on level $N$ if they can be connected by a path which does not intersect any vanishing curves on level $N$. If two regions are contiguous on level $N$, then the signature of $M_{N}$ is the same in both regions, because the signature can only change when a vanishing curve is crossed. For each $C_{p, q}$ on level $N$, for $p q \leqq N$, choose a neighborhood $U$ of $C_{p, q}$ small enough so that the only other vanishing curves on level $N$ which intersect $U$ also intersect $C_{p, q} . U-C_{p, q}$ has two connected components. Define the $c>1$ side of $C_{p, q}$ to be the connected component on the right of $C_{p, q}$, moving forward, if $p \geqq q$, and on the left, moving forward, if $p<q$. The other component is called the $c>1$ side of $C_{p, q}$. The motivation for this terminology is that the $c>1$ side of $C_{p, q}$, for $c$ near 1, is contiguous on level $N=p q$ with the region $c>1, h>0$. This is easily verified by expanding $h_{p, q}(c)$ around $\mathrm{c}=1$. It follows that $M_{N}(c, h)$ is a positive matrix on the $c>1$ side of $C_{p, q}$ for $c$ near 1. $\operatorname{det} M_{N}$ vanishes to first order on $C_{p, q}$. Therefore $\operatorname{det} M_{N}(c, h)$ is negative on the $c<1$ side of $C_{p, q}$, for $c$ near but not at 1 . The sign of $\operatorname{det} M_{N}(c, h)$ can only change at a vanishing curve, so $\operatorname{det} M_{N}(c, h)$ is negative in the entire region of the $c<1$ half-plane which is contiguous on level $p q$ to the $c<1$ side of $C_{p, q}$ for $c$ near but not at 1. By Proposition 5, this region is $R_{p, q}$. So the region $R_{p, q}$ is eliminated. The induction step now follows from Proposition 6.

Given Propositions 2 and 7, we are left with the task of eliminating the intervals on the curves $C_{p, q}^{\prime}$ in between the points in the discrete list (5). Let $I_{p, q, k}, k \geqq 2$, be the open interval on $C_{p, q}^{\prime}$ between $F_{p, q, k-1}$ and $F_{p, q, k}$. Let $I_{p, q, 1}$ be the open subset of $C_{p, q}^{\prime}$ beyond $F_{p, q, 1}$. That is, $I_{p, q, 1}$ is the open subset of $C_{p, q}^{\prime}$ with $m<p+q$. Clearly,

\section{Proposition 8.}

$$
C_{p, q}^{\prime}=\bigcup_{k \geqq 0} I_{p, q, k} \bigcup_{k \geqq 1} F_{p, q, k} .
$$

The goal is to eliminate the open intervals $I_{p, q, k}, k \geqq 1$. Recall that, when $C_{p^{\prime}, q^{\prime}}=C_{q+k-1, p+k}$ first appears on level $N^{\prime}=p^{\prime} q^{\prime}$, there is a negative metric state on its $c<1$ side, near $c=1$. We will show that this negative metric state continues to exist on the $c<1$ side of $C_{p^{\prime}, q^{\prime}}$ moving away from $c=1$, and in particular exists on $C_{p, q}^{\prime}$ on the $c<1$ side of $C_{p^{\prime}, q^{\prime}}$. That part of $C_{p, q}^{\prime}$ is a subset of $I_{p, q, k}$, and, by the definition of first intersections, there are no intersections on $I_{p, q, k}$ at level $N^{\prime}$. It will then follow that there is a negative metric state on all of $I_{p, q, k}$, and we will be done.

Proposition 9. On level $N^{\prime}=p^{\prime} q^{\prime}$, the first $k$ successive intersections on $C_{p^{\prime}, q^{\prime}}$, are with $C_{p+k-j, q+k-j}^{\prime}, 1 \leqq j \leqq k$. These are the first intersections $F_{p+k-j, q+k-j, j}$ on $C_{p+k-j, q+k-j}^{\prime}$, occurring at $m=p+q+2 k-j-1$.

Proof. The proof is straightforward algebra. 
Proposition 10. Suppose $\left(c^{\prime}, h^{\prime}\right)$ is on some $C_{p, q}, p q=N$, but is not on antersection of vanishing curves at level $N$. Then the null space on level $N$ is one dimensional at $\left(c^{\prime}, h^{\prime}\right)$.

Proof. $\operatorname{det} M_{N}(c, h)$ vanishes to first order at $C_{p, q}$ near $\left(c^{\prime}, h^{\prime}\right)$.

Proposition 11. At $F_{p, q, k}$, the intersection of $C_{p, q}^{\prime}$ and $C_{p^{\prime}, q^{\prime}}=C_{q-1+k, p+k}, k \geqq 1$, occurring at $c=c(m), h=h_{p, q}(c), m=p+q+k-1$,

$$
\operatorname{det} M_{p^{\prime} q^{\prime}-p q}(c, h+p q) \neq 0 \text {. }
$$

Proof. If this determinant were zero, then $(c, h+p q)$ would be on a vanishing curve $C_{r, s}$ on level $r s=p^{\prime} q^{\prime}-p q$. Direct calculation of $p^{\prime} q^{\prime}-p q$ gives

$$
r s=m(m+1)-(m+1) p-m q .
$$

The condition that $(c, h+p q)$ lie on $C_{r, s}$ is

$$
(m+1) p+m q= \pm((m+1) r-m s) \text {. }
$$

It follows from Eqs. $(10,11)$ that $r=m$ or $s=m+1$. But this gives a contradiction if we take Eq. (10) $\bmod m$ or $\bmod m+1$, since $1 \leqq p<m$ and $1 \leqq q<m+1$.

Proposition 12. For $j=1,2, \ldots, k$ there exists an open neighborhood $U_{p^{\prime}, q^{\prime}, j}$ of

$$
F_{p+k-j, q+k-j, j}=F_{q^{\prime}-j, p^{\prime}+1-j, j},
$$

and a nowhere zero analytic function $v_{j}(c, h)$, defined on $U_{p^{\prime}, q^{\prime}, j}$ with values in level $N^{\prime}=p^{\prime} q^{\prime}$ of $V(c, h)$, such that $v_{j}(c, h)$ is in the null space of level $N^{\prime}$ if and only if $(c, h)$ is on $C_{p^{\prime}, q^{\prime}}$.

Proof. Write $p^{\prime \prime}=p+k-j, q^{\prime \prime}=q+k-j, N^{\prime \prime}=p^{\prime \prime} q^{\prime \prime}<N^{\prime}$. Let $U=U_{p^{\prime}, q^{\prime}, j}$ be a neighborhood of $F_{p+k-j, q+k-j, j}$ small enough that it intersects no vanishing curves but $C_{p^{\prime \prime}, q^{\prime \prime}}^{\prime}$ and $C_{p^{\prime}, q^{\prime}}$ on level $N^{\prime}$. Choose coordinates $(x, y)$ in $U$, analytic in $(c, h)$ and real for $c, h$ real, such that $C_{p^{\prime \prime}, q^{\prime \prime}}^{\prime}$ is given by $x=0$ and $C_{p^{\prime}, q^{\prime}}$ is given by $y=0$. This is possible because the intersection is transversal. At level $N^{\prime \prime}, x=0$ is the only vanishing curve in $U$. The one dimensional null spaces of level $N^{\prime \prime}$ form a line bundle over the vanishing curve $x=0$ near $y=0$. Let $v_{j}^{\prime \prime}(0, y)$ be a nowhere zero analytic section of this line bundle, and let $v_{j}^{\prime \prime}(x, y)$ be an analytic function on $U$ with values in level $N^{\prime \prime}$, which extends this section. Define the subspace $V^{\prime \prime}(x, y)$ of level $N^{\prime}$ to be the span of the vectors

$$
L_{-k_{1}} L_{-k_{2}} \ldots L_{-k_{n}} v_{j}^{\prime \prime}(x, y), \quad 1 \leqq k_{1} \leqq k_{2} \leqq \ldots \leqq k_{n}, \quad \sum k_{i}=N^{\prime}-N^{\prime \prime} .
$$

The dimension of $V^{\prime \prime}(x, y)$ is $P\left(N^{\prime}-N^{\prime \prime}\right)$. For $y \neq 0$, the order of vanishing of $\operatorname{det} M_{N^{\prime}}(x, y)$ at $x=0$ is also $P\left(N^{\prime}-N^{\prime \prime}\right)$. Therefore, for $y \neq 0, V^{\prime \prime}(0, y)$ is the null subspace of level $N^{\prime}$. Let $V^{\prime}(x, y)$ be a subspace of level $N^{\prime}$ complementary to $V^{\prime \prime}(x, y)$, so level $N^{\prime}$ is $V^{\prime \prime} \oplus V^{\prime}$. The matrix of inner products $M_{N^{\prime}}$ can now be written in block diagonal form:

$$
M_{N^{\prime}}(x, y)=\left(\begin{array}{cc}
x Q(x, y) & x R(x, y) \\
x R(x, y)^{t} & S(x, y)
\end{array}\right),
$$


where $Q$ and $S$ are symmetric matrices. Three blocks of $M_{N^{\prime}}(x, y)$ are divisible by $x$, as in Eq. (13), because $V^{\prime \prime}(0, y)$ is in the null subspace of level $N^{\prime}$.

The key point now is that $Q(0,0)$ is non-degenerate. To see this, first note that, for $n>0$, the vector $L_{n} v_{j}^{\prime \prime}(0, y)=0$, since $L_{n} v_{j}^{\prime \prime}(0, y)$ is in the null subspace of level $N^{\prime}-n$, which is trivial. From this, and from the explicit basis (12) for $V^{\prime \prime}(x, y)$, we see that

$$
Q(x, y)=M_{p^{\prime} q^{\prime}-p^{\prime \prime} q^{\prime \prime}}\left(c, h+p^{\prime \prime} q^{\prime \prime}\right)+O(x),
$$

where $(c, h)$ corresponds to $(0, y)$ under the change of coordinates. Since $(0,0)$ is the first intersection $F_{p^{\prime \prime}, q^{\prime \prime}, j}$, Proposition (11) gives $\operatorname{det} Q(0,0) \neq 0$.

Since $\operatorname{det} Q(0,0) \neq 0, Q(x, y)$ is non-degenerate on all of $U$, if necessary replacing $U$ by a smaller neighborhood of $(0,0)$. Let $W$ be the matrix

$$
\left(\begin{array}{cc}
1 & -Q^{-1} R \\
0 & 1
\end{array}\right)
$$

and make the change of basis

$$
M_{N^{\prime}} \rightarrow W^{t} M_{N^{\prime}} W=\left(\begin{array}{cc}
x Q(x, y) & 0 \\
0 & T(x, y)
\end{array}\right) .
$$

Let $V^{\prime \prime \prime}(x, y)$ be the new complement to $V^{\prime \prime}(x, y)$, on which $T(x, y)$ is the inner product. The order of vanishing argument implies that $\operatorname{det} T(x, y)$ is nonzero for $y \neq 0$ and vanishes to first order at $y=0$. The one dimensional null space of $T(x, 0)$ is the null space of level $N^{\prime}$ for $x \neq 0$. At $x=y=0$, the one dimensional null space of $T(0,0)$ spans, with $V^{\prime \prime}(x, y)$, the $P\left(N^{\prime}\right)-P\left(N^{\prime \prime}\right)+1$ dimensional null subspace of level $N^{\prime}$. By the same argument which gave $v_{j}^{\prime \prime}(x, y)$, we can choose a nowhere zero analytic function $v_{j}(x, y)$ on $U$, with values in $V^{\prime \prime \prime}(x, y)$, such that $v_{j}(x, 0)$ is in the null space of $T(x, 0)$ and therefore in the null space of level $N^{\prime}$. Since $T(x, y)$ is nondegenerate for $y \neq 0, v_{j}(x, y)$ is not in the null space of level $N^{\prime}$ if $y \neq 0$.

Let $J_{p^{\prime}, q^{\prime}, j}, 1<j \leqq k$, be the open interval on $C_{p^{\prime}, q^{\prime}}$ between

$$
F_{p+k-j, q+k-j, j} \text { and } F_{p+k-j-1, q+k-j-1, j+1} \text {. }
$$

Let $J_{p^{\prime}, q^{\prime}, 1}$ be the open interval on $C_{p^{\prime}, q^{\prime}}$ lying between $c=1$ and $F_{p+k-1, q+k-1,1}$. Let $W_{p^{\prime}, q^{\prime}, j}, 1 \leqq j \leqq k$, be a neighborhood in the plane which intersects no vanishing curves on level $N^{\prime}$ except $J_{p^{\prime}, q^{\prime}, j}$. For $j>1$, require

$$
\begin{gathered}
J_{p^{\prime}, q^{\prime}, j} \subset U_{p^{\prime}, q^{\prime}, j-1} \cup W_{p^{\prime}, q^{\prime}, j} \cup U_{p^{\prime}, q^{\prime}, j}, \\
W_{p^{\prime}, q^{\prime}, j} \cap U_{p^{\prime}, q^{\prime}, j} \neq \emptyset, \quad W_{p^{\prime}, q^{\prime}, j} \cap U_{p^{\prime}, q^{\prime}, j-1} \neq \emptyset .
\end{gathered}
$$

For $j=1$ require only

$$
W_{p^{\prime}, q^{\prime}, 1} \cap U_{p^{\prime}, q^{\prime}, 1} \neq \emptyset \text {. }
$$

Proposition 13. For each $j, 1 \leqq j \leqq k$, there is a nowhere zero analytic function $w_{j}(c, h)$ on $W_{p^{\prime}, q^{\prime}, j}$ with values in level $N^{\prime}$ such that $w_{j}(c, h)$ is in the null space of level $N^{\prime}$ if and only if $(c, h)$ is on $J_{p^{\prime}, q^{\prime}, j}$. On the intersections of their neighborhoods of definition, 
$w_{j}=f_{j} v_{j}$, where $f_{j}$ is a nonzero function, and $w_{j}=g_{j} v_{j-1}$, where $g_{j}$ is a nonzero function.

Proof. Again, the null space of level $N^{\prime}$ is trivial on $W_{p^{\prime}, q^{\prime}, j}$ except on $J_{p^{\prime}, q^{\prime}, j}$, where it is one dimensional.

Proposition 14. The level $N^{\prime}$ metric is negative on the vectors $v_{p^{\prime}, q^{\prime}, j}(c, h)$ and on the vectors $w_{p^{\prime}, q^{\prime}, j}(c, h)$, on the $c<1$ side of $C_{p^{\prime}, q^{\prime}}$.

Proof. The matrix $M_{N^{\prime}}$ is positive in $W_{p^{\prime}, q^{\prime}, 1}$ on the $c>1$ side of $C_{p^{\prime}, q^{\prime}}$, by the contiguity argument, since there are no intersections on $C_{p^{\prime}, q^{\prime}}$ between $W_{p^{\prime}, q^{\prime}, 1}$ and $c=1$. The inner product is thus positive on $w_{p^{\prime}, q^{\prime}, 1}$ on the $c>1$ side of $C_{p^{\prime}, q^{\prime}}$. The inner product vanishes to first order on $w_{p^{\prime}, q^{\prime}, 1}$ on $C_{p^{\prime}, q^{\prime}}$. Therefore the inner product is negative on $w_{p^{\prime}, q^{\prime}, 1}$ on the $c<1$ side of $C_{p^{\prime}, q^{\prime}}$. The proposition now follows by induction on the series $w_{1}, v_{1}, w_{2}, v_{2}, \ldots$, since neighboring vectors in the series differ by nonzero functions $f_{j}$ or $g_{j}$, and since the $w_{j}(c, h)$ and $v_{j}(c, h)$ are in the level $N^{\prime}$ null space only for $(c, h)$ on $C_{p^{\prime}, q^{\prime}}$.

Proposition 15. $I_{p, q, k}$ is eliminated on level $N^{\prime}=(q+k-1)(p+k)$.

Proof. By the previous proposition, the metric is negative on $v_{p^{\prime}, q^{\prime}, k}(c, h)$, on the $c<1$ side of $C_{p^{\prime}, q^{\prime}}$. But $I_{p, q, k}$ approaches arbitrarily close to $C_{p^{\prime}, q^{\prime}}$ on the $c<1$ side within $U_{p^{\prime}, q^{\prime}, k}$. Therefore $M_{N}(c, h)$ has a negative eigenvalue at one end of $I_{p, q, k}$. But the signature of $M_{N}(c, h)$ cannot change along $I_{p, q, k}$, because there are no intersections at level $N^{\prime}$ on $I_{p, q, k}$. The proposition follows.

Propositions 2, 7, 8, and 15 imply Theorem 1.

Acknowledgement. We thank Adrian Kent for a critical reading of the manuscript.

Note added in proof. A similar but not identical version of the details of the non-unitarity proof has been given by Langlands [10].

\section{References}

1. Friedan, D., Qiu, Z., Shenker, S.: In: Vertex operators in mathematics and physics. Lepowsky, J. et al. (eds.), Berlin, Heidelberg, New York: Springer 1984, p. 419; Conformal invariance, unitarity and critical exponents in two dimensions. Phys. Rev. Lett. 52, 1575 (1984)

2. Friedan, D., Qiu, Z., Shenker, S.: Superconformal invariance in two dimensions and the tricritical Ising model. Phys. Lett. 151B, 37 (1985)

3. Goddard, P., Olive, D.: Kac-Moody algebras, conformal symmetry and critical exponents. Nucl. Phys. B 257, 83 (1985); Goddard, P., Kent, A., Olive, D.: Virasoro algebras and coset space models. Phys. Lett. 152B, 88 (1985); Unitary representations of the Virasoro and superVirasoro algebras. Commun. Math. Phys. 103, 105 (1986)

4. Boucher, W., Friedan, D., Kent, A.: Determinant formulae and unitarity for the $N=2$ superconformal algebras in two dimensions or exact results in string compactification. Phys. Lett. 172B, 316 (1986)

5. Boucher, W., Friedan, D., Kent, A., Shenker, S.: In preparation

6. Di Vecchia, P., Petersen, J.L., Yu, M., Zheng, H.B.: Explicit construction of unitary representations of the $N=2$ superconformal algebra. Phys. Lett. 174B, 280 (1986) 
7. Kac, V.: Lecture Notes in Physics, Vol. 94, p. 441. Berlin, Heidelberg, New York: Springer 1979; in Proc. Int. Congress of Math., Helsinki (1978)

8. Feigin, B.L., Fuchs, D.B.: Funct. Anal. Appl. 16, 114 (1982)

9. Kac, V.: Lecture Notes in Mathematics, Vol. 933, p. 117. Berlin, Heidelberg, New York: Springer 1982

10. Langlands, R.P.: On unitary representations of the Virasoro algebra. In: Proceedings of the Montreal Workshop on infinite dimensional Lie algebras and their applications. Kass, S. (ed.) (to appear)

Communicated by A. Jaffe

Received May 23, 1986 